approach, engaging in opportunistic and ongoing discussions concerning malignant and non-malignant disease.

Clinicians who engaged patients and their families in multiple conversations about their needs and treatments, and who sought to include psychological, social as well as biomedical aspects of care, report providing this aspect of end of life care as integral to providing compassionate care at the end of life.

\title{
P 203 INITIATING DISCUSSION IN END OF LIFE CARE: CLINICIAN'S PERSPECTIVES
}

Kerry Jones, ${ }^{1}$ Rebecca Baines, ${ }^{2}$ lain Lang'. ' University of Exeter Medical School, Exeter, United Kingdom; ${ }^{2}$ Hospiscare, Exeter, United Kingdom

10.1136/bmjspcare-2014-000654.244

Background and Aims Engaging patients nearing the end of life in discussion about their subsequent treatment is regarded as a vital aspect to the provision of effective and cost-effective palliative care (Department of Health, 2011; National Institute of Clinical Excellence, 2012). There is little research to date which suggests how such discussions can be easier to initiate or how clinicians can optimise their communication with patients and their families on end of life care.

These issues are typically addressed using Advance Care Plans such as the Liverpool Care Pathway or a locally developed Treatment Escalation Plan (TEP) and a multi professional framework for the delivery of compassionate care in the last hours and days of life. The TEP was developed in response to variability in care planning and the lack of patient and carer involvement in it.

In this paper, I discuss clinician's accounts of the barriers and facilitators to initiating conversations about advanced care planning at the end of life with patients and their families and their approaches to overcoming these challenges.

Methods and Results Data collected from 20 qualitative in-depth interviews with Palliative Care Consultants and other senior clinician's in a hospice and hospital environment show that several domains were identified as vital to the provision of a compassionate approach to end of life care. These include, developing a trusting and ongoing relationship with both the patient and family members, adopting a multi disciplinary 